1

2

3

4

5

6

7

8

9

10

11

12

13

14 12

\title{
Optimizing oleaginous yeast cell factories for flavonoids and hydroxylated flavonoids biosynthesis
}

3

Yongkun Lv ${ }^{1,2,4}$, Mattheos Koffas ${ }^{3}$, Jingwen Zhou ${ }^{2,4^{*}}$ and Peng Xu ${ }^{1^{*}}$ 5

${ }^{1}$ Department of Chemical, Biochemical and Environmental Engineering, University of Maryland Baltimore County, Baltimore, MD 21250

${ }^{2}$ National Engineering Laboratory for Cereal Fermentation Technology, Jiangnan University, 1800 Lihu Road,

${ }^{3}$ Department of Chemical and Biological Engineering, Rensselaer Polytechnic Institute, Troy, NY 12180

${ }^{4}$ Jiangsu Provisional Research Center for Bioactive Product Processing Technology, Jiangnan University, 1800 Lihu

Road, Wuxi, Jiangsu 214122, China.

* Corresponding author Tel: +1(410)-455-2474; fax: +1(410)-455-1049.

E-mail address: pengxu@umbc.edu (Peng Xu); zhoujw1982@jiangnan.edu.cn (Jingwen Zhou) 


\section{Abstract}

Plants possess myriads of secondary metabolites with a broad spectrum of health-promoting benefits. Up to date, plant extraction is still the primary route to produce high-value natural products, which inherently suffers from economics and scalability issues. Heterologous production in microbial host is considered as a feasible approach to overcoming these limitations. Flavonoid and its hydroxylated derivatives represent a diversified family of bioactive compounds, most prominently known as antioxidant and anti-aging agents. Oleaginous yeast is rich in hydrophobic lipid bodies and spatially-organized organelles, which provides the ideal environment for the regioselectivity and stereoselectivity of many plant-specific enzymes. In this report, we validated that $Y$. lipolytica is a superior platform for heterologous production of high-value flavonoids and hydroxylated flavonoids. By modular construction and characterization, we determined the rate-limiting steps for efficient flavonoids biosynthesis in $Y$. lipolytica. We evaluated various precursor pathways and unleashed the metabolic potential of $Y$. lipolytica to produce flavonoids, including the supply of acetyl-CoA, malonyl-CoA and chorismate. Coupled with the optimized chalcone synthase module and the hydroxylation module, our engineered strain produced $252.4 \mathrm{mg} / \mathrm{L}$ naringenin, $134.2 \mathrm{mg} / \mathrm{L}$ eriodictyol and $110.5 \mathrm{mg} / \mathrm{L}$ taxifolin from glucose. Collectively, these findings demonstrate our ability to harness oleaginous yeast as microbial workhorse to expand nature's biosynthetic potential, enabling us to bridge the gap between drug discovery and natural product manufacturing.

Keywords: Natural products, Flavonoids, Hydroxylation, Metabolic engineering, Oleaginous yeast

Running title: Engineer oleaginous yeast factory for flavonoids

\section{Introduction}

Plant possess myriads of secondary metabolites with a broad spectrum of health-promoting benefits. Plant-derived natural products (PNPs) have been used to suppress tumor growth, inhibit retrovirus replication, treat metabolic disease and modulate cholesterol level in both animal and human tests ${ }^{1}$. Up to date, plant extraction is still the primary route to produce PNPs

1. However, isolation of PNPs from their native sources is limited by low abundance and environmental, seasonal, and regional variations. Total chemical synthesis of complex PNPs often involves toxic catalyst and is commercially unsustainable due to low yield, safety concerns and strict GMP regulations ${ }^{2}$. Genome-mining of plant metabolic pathways ${ }^{3-8}$ and reconstruction of biosynthetic gene clusters (BGCs) in industrially-relevant microbes offer significant promise for discovery and scalable synthesis of plant natural products.

E. coli and $S$. cerevisiae have long been established as host strains to manufacture a large 
50 variety of plant natural products ${ }^{3,7-14}$. The recent development of oleaginous yeast platforms 51 offers significant advantages over E. coli and $S$. cerevisiae (Table 1). The high precursor 52 acetyl-CoA and malonyl-CoA flux along with the hydrophobic lipid bodies make oleaginous 53 yeast a promising host to produce various natural products with complex structures. For example, 54 Y. lipolytica is known to internalize substantial portion of carbon feedstock as lipids and fatty acids ${ }^{15,16}$, which provides the ideal amphiphilic environment for the catalytic function of many plant-derived enzymes. It has been recognized as a 'generally regarded as safe' (GRAS) organism for the production of organic acids, polyunsaturated fatty acids (PUFAs) ${ }^{17,18}$ and carotenoids ${ }^{19-22}$ in the food and nutraceutical industry. Compared to S. cerevisiae, Y. lipolytica lacks Crabtree effects, which doesn't require the co-feeding of ethanol for cell to grow. The low $\mathrm{pH}$ tolerance ${ }^{23}$, strictly aerobic nature ${ }^{24,25}$ and versatile substrate-degradation profile ${ }^{25-27}$ enable its robust growth from a wide range of renewable feedstocks. Genetic toolbox development has been expanding to protein expression ${ }^{28-30}$, promoter characterization ${ }^{31-33}$, YaliBrick-based cloning 34, 35, Golden gate cloning 21, 36, Piggyback transposon 37, genome-editing 34,38,39 and iterative gene integration ${ }^{40}$, affording us a collection of facile genetic tools for streamlined and accelerated pathway engineering in oleaginous yeast species.

Flavonoids represent a diversified family of phenylpropanoid-derived plant secondary metabolites, with an estimated 10,000 unique structures ${ }^{41,42}$. They are widely found in fruits, vegetables and medicinal herbs and plants. Pharmaceutical studies and animal tests have demonstrated their anti-obesity, anti-cancer, anti-inflammatory, and anti-diabetic activities ${ }^{43,44}$. Flavonoids are among the phytochemicals with proven activity towards the prevention of aging-related diseases, including the treatment of nervous and cardiovascular diseases, Parkinson's and Alzheimer's disease etc. ${ }^{45}$. These health-promoting benefits make flavonoids a distinct family of molecules to fight aging in personal care, nutraceutical industry and clinical trials. Considering the worldwide population with age older than 65 will triple in next 30 years (data from WHO, world health organization), there will be increasing interests and sustainable market demand globally in the near future.

To prepare the technological ground and enable microbial synthesis, various flavonoid pathways have been reconstituted in various microbial species including E. coli and S. cerevisiae recently, and the recombinant production of an array of molecules such as naringenin 46,47 , eriodyctiol ${ }^{48,49}$, resveratrol ${ }^{50-54}$, pinocembrin ${ }^{49,55}$, anthocyanins ${ }^{56-58}$, quercetin, kaempferol ${ }^{59}$, 81 silybin, isosilybin ${ }^{60}$, baicalein and scutellarein ${ }^{61}$ has been described. Most of the reported 82 studies were centered around simple flavanones or chalcones through enzymatic cascade reactions with the feeding of expensive phenyl precursors (phenylalanine, tyrosine, $p$-coumaric acid and caffeic acid etc), which limits our opportunity for scalable and low-cost production. Structural activity relationship (SAR) studies demonstrate the side chain modifications are highly correlated with flavonoid biological activities 42, 62. The hydroxylation of flavonoids improves their metabolic stability and membrane permeability, and enhances the solubility and 
89 hydroxylated flavonoids, due to our limited ability to functionally express plant P450

90 hydroxylases and the cytochrome P450 reductases ${ }^{64}$. Oleaginous yeast is rich in membrane 91 structure and subcellular compartments (i.e. lipid bodies, peroxisome, ER and oleosome), which 92 provides the hydrophobic environment that is critical for regioselectivity and stereoselectivity in 93 hydroxylation, glycosylation and prenylation of flavonoids ${ }^{65-68}$. Developing a P450 expression 94 platform in oleaginous yeast will enable us to access the vast majority of complex natural 95 products and deliver robust microbial cell factories to meet the market demand.

96 To bridge this gap, we tested and assessed various plant-derived polyketide synthases, P450 monooxygenase/hydroxylases and cytochrome P450 reductases in Y. lipolytica, to diversify the structure of flavonoids. With naringenin, eriodyctiol and taxifolin as testing molecules, we characterized the catalytic efficiency of various plant enzymes, including tyrosine ammonia lyase (TAL), 4-coumaroyl-CoA ligase (4CL), chalcone synthase (CHS), chalcone isomerase (CHI), flavonone-3'-hydroxylase (F3'H), flavonol-3-hydroxyalse $(\mathrm{F} 3 \mathrm{H})$ and cytochrome $\mathrm{P} 450$ reductases. These plant-derived genes were coexpressed with the endogenous acetyl-CoA carboxylase (ACC1) and the pentafunctional AROM polypeptide (ARO1). Systematic pathway debottlenecking indicates that chalcone synthase, $\mathrm{ACC} 1$ and cytochrome $\mathrm{P} 450$ reductases are the rate-limiting steps for hydroxylated flavonoid production. Specifically, increasing PhCHS copy number and controlling culture $\mathrm{pH}$ elevated naringenin production up to $252.4 \mathrm{mg} / \mathrm{L}$. Screening four cytochrome P450 reductases led us to identify that CrCPR derived from Catharanthus roseus is the most efficient electron shuttle to complete the hydroxylation reaction, despite that endogenous ylCPR1 (YALI0D04422g) displays similar function with relatively low efficiency.

110 Further expression of the plant-derived P450 enzymes, including the flavanol-3' hydroxylase

111 (GhF3'H) from Gerbera hybrid led the engineered strain to produce about $110.5 \mathrm{mg} / \mathrm{L}$ of 112 taxifolin and $134.2 \mathrm{mg} / \mathrm{L}$ of eriodictyol. This work set the foundation for us to engineer 113 oleaginous yeast as chassis for cost-efficient production of flavonoids and hydroxylated

114 flavonoids. The functional expression of plant-derived polyketide synthase, P450 115 monooxygenase and reductases will expand our capability to access nature's biosynthetic 116 potential for drug discovery and natural product manufacturing.

\section{Results and discussion}

\section{Modular construction and characterization of flavonoid pathway in $\mathbf{Y}$. lipolytica}

119 The availability of intracellular malonyl-CoA was reported to be a rate-limiting step of 120 flavonoid synthesis in many microorganisms ${ }^{69-71}$. Considering the high acetyl-CoA and 121 malonyl-CoA flux, we firstly reconstructed the synthetic pathway and validated the feasibility of 122 using $Y$. lipolytica as the chassis to produce flavonoids. In addition, the cytochrome $c$ P450 123 (CYP) flavonoid 3'-hydroxylase (F3'H) plays a critical role in oxidizing the phenyl ring and 124 generating hydroxylated flavonoids ${ }^{72}$. Based on the distribution of potential rate-limiting steps, 125 we rationalized and partitioned the flavonoids pathway into two modules, the naringenin 
126 synthesis module (Module I) and the hydroxylation module (Module II) (Fig. 1). Module I 127 contains essential precursor pathway to provide shikimic acid, malonyl-CoA and chalcone 128 precursors; while Module II contains the cytochrome $c$ P450 (CYP) flavonoid 3'-hydroxylase 129 (F3'H) and cytochrome $c$ P450 reductase (CPR). As a direct assessment of the module efficiency, 130 we have established HPLC method to analyze naringenin, eriodyctiol and taxifolin (Fig. 2).

131 Naringenin is the starting point for many flavonoid functionalization chemistry. We first constructed Module I in Y. lipolytica Polf to synthesize chalcone and naringenin. Because genes from different plants have different specificity and activity, we selected two genes for each of

134 the first three steps in Module I based on the sequence alignment of closely-related plant species. 135 Pathways containing 4CL ( $p$-coumaric acid-CoA ligase), CHS (chalcone synthase) and CHI 136 (chalcone isomerase) were assembled in monocistronic forms by YaliBricks cloning platform ${ }^{34}$. 137 We observed that all eight constructs containing 4CL, CHS and CHI resulted in the synthesis of 138 naringenin from $p$-coumaric acid, with production ranging from $10 \mathrm{mg} / \mathrm{L}$ to $21.5 \mathrm{mg} / \mathrm{L}$ (Fig. 3a). 139 Interestingly, the three top producers (Fig. 3a) share the same source of chalcone synthase from 140 Petunia $x$ hybrid, indicating that chalcone synthase dictates the efficiency of Module I. To 141 achieve de novo synthesis of naringenin, we further introduced tyrosine ammonia-lyase (RtTAL) 142 from Rhodotorula toruloides, which has been reported to generate phenylpropanoid precursors 143 from glucose ${ }^{47,73}$. With the overexpression of RtTAL, we detected $p$-coumaric acid as the direct 144 de-amination product of tyrosine (Supplementary Fig. S1). By complementing the 145 4CL-CHS-CHI pathway, the resulted strain $Y$. lipolytica Po1f/T4SI produced $14.9 \mathrm{mg} / \mathrm{L}$ 146 naringenin from glucose (Supplementary Fig. S1). These results validated the feasibility of using $147 \quad$ Y. lipolytica as chassis for de novo synthesis of naringenin.

148 There has been a number of reports that $Y$. lipolytica could selectively hydroxylate limonene to perillyl alcohol, perillaldehyde and perillic acids ${ }^{74,75}$, demonstrating the endogenous P450

150 monooxygenase and cytochrome P450 reductase is active enough to hydroxylate methyl group 151 on monoterpenes. Our lab has demonstrated the functional expression of the P450 152 monooxygenase that selectively hydroxylates protodeoxyviolaceinic acid to protoviolaceinic 153 acid, generating the greenish pigment in Y lipolytica $^{76}$. On the basis of these results, we argue 154 that Y. lipolytic could be an excellent platform for expression of plant P450 enzymes.

$155 \quad \mathrm{~F}^{\prime} \mathrm{H}$ is the critical enzyme involved in the functional hydroxylation of flavonoids. 156 Cytochrome $c$ P450 reductase (CPR) is required for electron transfer from NADPH to CYP ${ }^{77}$. 157 We have chosen two plant-derived F3'Hs and three CPRs to evaluate which F3'H-CPR pairs 158 could perform hydroxylation chemistry (Fig. 3b). All 6 combinations of F3'H-CPR pairs 159 produced eriodictyol. We observed that strain Polf/HR with overexpression of CrCPR (derived 160 from Catharanthus roseus ${ }^{78}$ coupled with GhF3'H (derived from Gerbera hybrid) or GmF3'H 161 (derived from Glycine max), led to the highest eriodictyol production around $39 \mathrm{mg} / \mathrm{L}$, with 162 molar conversion yield up to $73.7 \%$ from naringenin (Fig. 3b). Interestingly, the two 163 yeast-sourced CPRs, Y1CPR from Y. lipolytica and ScCPR from S. cerevisiae S288c, also gave 
164 rise to eriodictyol, indicating the endogenous CPR is sufficient to shuttle electrons from 165 NADPH to the active oxygen species, which is consistent with the findings reported by Leonard 16679 . We further constructed strain Y. lipolytica Po1f/HRH with the overexpression of flavanone 167 3-hydroxylase (SIF3H from Solanum lycopersicum) and detected about $26.0 \mathrm{mg} / \mathrm{L}$ taxifolin with 168 a molar yield of $46.5 \%$ from naringenin (Fig. 2). In addition, Y. lipolytica endogenous CPR 169 matched F3'H well, with comparable efficiency as CrCPR (Fig. 3b), which is consistent with 170 previous report that $Y$. lipolytica was capable of performing P450-based biotransformation ${ }^{80,81}$. 171 To achieve de novo synthesis of eriodictyol and taxifolin, we further complemented the RtTAL 172 with GhF3'H and SIF3H, resulting in strains Po1f/T4SIHR and Po1f/T4SIHRH, respectively. 173 When these strains were tested in shake flask cultures, we obtained $17.2 \mathrm{mg} / \mathrm{L}$ eriodictyol and $17411.3 \mathrm{mg} / \mathrm{L}$ taxifolin from glucose, respectively. These results validated that $Y$. lipolytica will be 175 an ideal chassis to functionally express plant P450 enzymes and produce hydroxylated 176 flavonoids.

177 Tuning gene-copy number to remove pathway bottlenecks

178 The balance of metabolic flux and mitigation of metabolic burden is a vital factor for 179 optimizing metabolite production in microorganisms 9, 53, 82. Introduction of large gene cluster 180 may result in the host strain losing cellular fitness when the expression of heterologous proteins 181 exceeds the carrying capacity of the system. For example, metabolic flux improvement by 182 overexpression of upstream pathways may not be accommodated by downstream pathways ${ }^{82}$; 183 intermediate accumulation or depletion may reduce cell viability ${ }^{83}$; and overexpressed gene 184 clusters may overload the cell and elicit cellular stress response ${ }^{84,85}$. We next attempted to probe 185 the rate-limiting steps in Module I and Module II by gradually increasing gene copy number of 186 the genes involved. Gene copy number of each enzymatic step was individually tuned by using 187 YaliBrick assembly platform ${ }^{34}$. Naringenin production increased by 2.64 -fold when the gene 188 copy number for chalcone synthase (PhCHS) increased from one to five (Fig. 4a), indicating that 189 CHS is the rate-limiting step in Module I. Increasing the gene copy number of other metabolic 190 genes (RtTAL and Pc4CL) did not have obvious effect on naringenin titer, while increasing the 191 gene copy number of $\mathrm{MsCHI}$ decreased naringenin titer by 34.4\% (Fig. 4a). We determined that 192 the optimal gene copy number for $\mathrm{PhCHS}$ is 5, as naringenin production was only marginally 193 increased when the gene copy number was changed from 4 to 5 . As larger plasmid may cause 194 genetic instability, we did not further increase the copy number of PhCHS.

195 In Module II, increasing one copy number of $C r C P R$ resulted in eriodictyol and taxifolin 196 titers increasing by $26.8 \%$ and $22.3 \%$, reaching $48.1 \mathrm{mg} / \mathrm{L}$ and $31.8 \mathrm{mg} / \mathrm{L}$ (Fig. 4b), respectively. 197 Increasing the copy number of $S I F 3 H$ and $G h F 3$ ' $H$ did not have obvious effect on eriodictyol or 198 taxifolin production (Fig. 4b), indicating that CPR is the rate-limiting step in Module II. 199 Eriodictyol and taxifolin titers remained stable, when the gene copy number for $C r C P R$ was 200 increased from 2 to 5, suggesting that the optimal ratio of F3' $\mathrm{H}$ to its reductase $C r C P R$ is $1: 2$, 201 which is consistent with previous report ${ }^{86}$. The naringenin-to-eriodictyol and taxifolin 
202

203

204

205

206

207

208

209

210

211

212

213

214

215

216

217

218

219

220

221

222

223

224

225

226

227

228

229

230

231

232

233

234

235

236

237

238

239

conversion ratio reached $90.5 \%$ and $56.8 \%$, respectively (Fig. 4b), under the optimal F3'H-CPR ratios. To achieve de novo synthesis of eriodictyol and taxifolin, we complemented the eriodictyol and taxifolin pathways with the RtTAL pathway. The resulting strains Po1f/T4S ${ }_{x 5} I_{H R} 2$ and Po1f/T4S ${ }_{x} I_{\mathrm{IHR}} \mathrm{H}$ produced $28.9 \mathrm{mg} / \mathrm{L}$ eriodictyol and $25.2 \mathrm{mg} / \mathrm{L}$ taxifolin from glucose, respectively. These titers are $68.0 \%$ and $123.0 \%$ higher than the control strains Po1f/T4SIHR and Po1f/T4SIHRH. These results confirmed that tuning gene copy numbers will be a critical step to remove pathway bottlenecks and achieve metabolic balance in genetically modified cell factories, in particularly, oleaginous yeast for flavonoids production.

\section{Improving flavonoid production by enhancing precursor synthesis}

We next sought to investigate the upstream shikimic acid and malonyl-CoA pathways to further improve flavonoids production. By supplementing $100 \mathrm{mg} / \mathrm{L}$ L-tyrosine with the strain Polf/T4SI, we observed that naringenin production was increased by $33.6 \%$ with glucose as sole carbon source, indicating that upstream shikimic acid pathway is a bottleneck for naringenin synthesis in Y. lipolytica. We then overexpressed the pentafunctional polypeptides arom protein ARO1, which catalyzes steps 2 through 6 in the biosynthesis of chorismate, to boost the precursor for L-tyrosine synthesis ${ }^{87}$. YALI0F12639g (Y1ARO1) is a Y. lipolytica homologue of S. cerevisiae ARO1 ${ }^{88}$, and the DNA sequence for YlARO1 is composed of 1 intron and 2 exons, encoding a 1556-aa protein. To mitigate unintended mRNA splicing and transcriptional regulation, we removed the internal intron for YlARO1. When this YlARO1 gene was overexpressed in strain Po1f/T4S $\mathrm{S}_{\mathrm{x}} \mathrm{I}$ with optimal Module I settings, naringenin production was increased to $81.6 \mathrm{mg} / \mathrm{L}$, a $50.9 \%$ increased compared to the parental strain (Fig. 5a). When we combined Module I with Module II, the resulting strains Po1f/AT4S $\mathrm{x}_{5} \mathrm{IHR}_{\mathrm{x} 2}$ and Po1f $/ \mathrm{AT}_{4} \mathrm{~S}_{\mathrm{x} 5} \mathrm{IHR}_{\mathrm{x} 2} \mathrm{H}$ produced $40.1 \mathrm{mg} / \mathrm{L}$ eriodictyol and $33.4 \mathrm{mg} / \mathrm{L}$ taxifolin, which is $38.8 \%$ and $32.5 \%$ higher than that of the control strains Po1f/T4S $\mathrm{S}_{\mathrm{x}} \mathrm{IHR}_{\mathrm{x} 2}$ and Po1f/T4S $\mathrm{S}_{\mathrm{x} 5} \mathrm{IHR}_{\mathrm{x} 2} \mathrm{H}$, respectively (Fig. 5b).

Acetyl-CoA and malonyl-CoA are shared precursors for both lipids and flavonoid pathway ${ }^{89,90}$. However, malonyl-CoA is primarily used to synthesize lipids cell membrane and support cell growth, leaving only a small amount of acetyl-CoA and malonyl-CoA for heterologous production. To mitigate this competition, it is desirable to redirect the acetyl/malonyl-CoA flux from lipid pathway to flavonoid pathway. Acetyl-CoA carboxylase (ACC) converts acetyl-CoA to malonyl-CoA, which is the first committed step in both lipid and flavonoids biosynthesis ${ }^{69}$. In order to enhance intracellular malonyl-CoA synthesis, we screened and tested different ACCs from three sources, including gram-positive bacteria Corynebacterium glutamicum ATCC 13032 (CgACC), gram-negative bacteria Escherichia coli MG1655 (Ec_accABCD), and Y. lipolytica (YlACC1, GRYC ID: YALI0C11407g) 91, 92. Biotin-apoprotein ligase modifies ACC by covalently attaching biotin, which is essential for ACC activity 93. EcBirA and YlBPL1 (YALI0E30591g) are E. coli and Y. lipolytica homologues of biotin-apoprotein ligase, respectively ${ }^{94,95}$. Genes encoding $\mathrm{CgACC}$, Ec_accABCD, and Y1ACC1, together with their 
240

241

242

243

244

245

246

247

248

249

250

251

252

253

254

255

256

257

258

259

260

261

262

263

264

265

266

267

268

269

270

271

272

273

274

275

276

277

biotin-apoprotein ligases were introduced to the naringenin-producing strain. All three ACCs could lead to substantial improvement in naringenin production (Fig. 5a), with YlACC1 demonstrating most obvious effect. For example, overexpression of YlACC1 in Po1f/AT4S ${ }_{x 5} I$ improved naringenin titer by $61.4 \%$, reaching $131.7 \mathrm{mg} / \mathrm{L}$ (Fig. 5a). The coupling of $E c \_a c c A B C D$ with EcBirA also resulted in naringenin production increasing by $22 \%$ compared with the strain without EcBirA overexpression, indicating the essential role of biotinylation in bacterial ACC activity. This is the first report that EcACC could be functionally expressed in oleaginous species. Unlike the bacterial ACC, co-expression of YlACC1 and YlBPL1 resulted in decreased naringenin production (Fig. 5a). This might indicate the endogenous biotin-apoprotein ligase (Y1BPL1) is sufficient to biotinylate ylACC1 in Y. lipolytica.

We observed that $\mathrm{pH}$ value dropped dramatically during the fermentation process (i.e. $\mathrm{pH}$ below 3.5 at the end of flask cultivation), and this could be largely ascribed to the overflown metabolism of TCA cycle and respiration ${ }^{20}$. It was recently discovered that acetate secretion was associated with the CoA-transfer reaction between acetyl-CoA and succinate in Y. lipolytica, encoded by a mitochondrial enzyme ylACH1 (YALI0E30965) ${ }^{23}$. To recycle acetate, we next sought to overexpress acetyl-CoA synthetases and convert acetate to acetyl-CoA. We tested three acetyl-CoA synthetases from E. coli, S. cerevisiae and Y. lipolytica (Fig. 5a). The native version $Y l A C S 2$ demonstrate better effect to recycle acetate. To test the combinatory effects of enhancing chorismate and acetyl/malonyl-CoA precursors, we overexpressed YlARO1 along with Y1ACS2-Y1ACC1 in strain Po1f/T4S ${ }_{x}$ I. The resulting strain produced $149.5 \mathrm{mg} / \mathrm{L}$ naringenin, which was $176.3 \%$ higher than the titer of the parental strain (Po1f/T4S $\mathrm{x}_{\mathrm{x}} \mathrm{I}$ ). We further applied the same strategy to Module II and tested whether overexpression of ARO1, ACC1 and ACS would benefit the accumulation of hydroxylated flavonoids. Overexpression of YlARO1 increased eriodictyol and taxifolin production by $38.8 \%$ and $32.5 \%$, yielding $40.1 \mathrm{mg} / \mathrm{L}$ and 33.4 mg/L (Fig. 5b), respectively. Overexpressing YlACS2 and YlACC1 further increased eriodictyol and taxifolin titers by $41.9 \%$ and $52.1 \%$, reaching $56.9 \mathrm{mg} / \mathrm{L}$ and $50.8 \mathrm{mg} / \mathrm{L}$, respectively (Fig. $5 \mathrm{~b}$ ). Due to the large size of the plasmid construct (more than $40 \mathrm{~kb}$ ), we did not further pursue the synergistic effect of ARO1, ACC1 and ACS2 in the current work. These results indicated that manipulation of acetyl-CoA, malonyl-CoA and chorismate pathway was critical to improve flavonoid production in $Y$. lipolytica.

\section{Boosting flavonoid production by bioprocess optimization}

The $\mathrm{C} / \mathrm{N}$ ratio is an important factor for regulating the acetyl-CoA and NADPH fluxes in Yarrowia lipolytica ${ }^{33}$. It has been reported that nitrogen starvation triggers the repression of TCA cycle and induces lipogenesis in oleaginous species ${ }^{96,97}$. It was recently discovered that $\mathrm{C} / \mathrm{N}$ ratio dynamically regulates lipogenic promoter activity in Y lipolytica $^{33}$. In this study, $\mathrm{C} / \mathrm{N}$ ratio was optimized in two patterns to improve flavonoid synthesis, by either adjusting the amount of nitrogen source (ammonia sulfate) or carbon source (glucose). The results showed that altering $\left(\mathrm{NH}_{4}\right)_{2} \mathrm{SO}_{4}$ content did not have obvious effect on naringenin titer. Slightly higher 
naringenin titer was achieved at higher $\mathrm{C} / \mathrm{N}$ ratio $(\mathrm{C} / \mathrm{N}=120)$ (Supplementary Fig. S2a). On the contrary, it was clearly shown that higher $\mathrm{C} / \mathrm{N}$ ratio was advantageous to improving naringenin titer by increasing the level of glucose. Specifically, naringenin titer was increased about $56 \%$ when the $\mathrm{C} / \mathrm{N}$ was altered from 40 to 160 (Supplementary Fig. S2b). Since glucose is the direct precursor for chorismate and malonyl-CoA, it indicates that there is still much space to further improve the precursor flux in $Y$. lipolytica.

In order to produce flavonoids with inexpensive YPD (yeast extract, peptone and dextrose) medium, we integrated the optimized pathways into Y. lipolytica Polf genome with our recently developed integration methods ${ }^{98}$. The best-performing strains NarPro/ASC, ErioPro, and TaxiPro produced $71.2 \mathrm{mg} / \mathrm{L}$ naringenin, $54.2 \mathrm{mg} / \mathrm{L}$ eriodictyol, and $48.1 \mathrm{mg} / \mathrm{L}$ taxifolin in YPD medium, respectively ${ }^{40}$. We observed that the $\mathrm{pH}$ dropped to 3.2 at the end of the fermentation in YPD, possibly due to the accumulation of various organic acids ${ }^{20}$. We next sought to buffer the media $\mathrm{pH}$ by using either phosphate buffer saline (PBS) or calcium carbonate $\left(\mathrm{CaCO}_{3}\right)$. Supplementation of $4 \% \mathrm{CaCO}_{3}$ maintained stable $\mathrm{pH}$ and improved naringenin titer by $31.2 \%$, reaching $138.1 \mathrm{mg} / \mathrm{L}$ at $144 \mathrm{~h}$, while PBS buffer did not have obvious effect compared with the control (Supplementary Fig. S4). We also analyzed the combinatory effects of inhibiting fatty acid synthesis by adding cerulenin ${ }^{99}$, maintaining stable $\mathrm{pH}$, and supplying sodium acetate. The fermentation time course showed that we could achieve steady improvement in naringenin production (Supplementary Fig. S5). For example, supplementation of $1 \mathrm{mg} / \mathrm{L}$ cerulenin with 40 $\mathrm{g} / \mathrm{L} \mathrm{CaCO}_{3}$ further improved naringenin titer by 31.2\%, (Supplementary Fig. S5c). However, supplying $5 \mathrm{mM} \mathrm{NaAc}$ did not result in further increase in naringenin production (Supplementary Fig. S5d). By intermittently feeding glucose after 48 hours, the chromosomally-integrated strain produced $252.4 \mathrm{mg} / \mathrm{L}$ naringenin under optimal conditions (Fig. 6a). Likewise, we tested the eriodictyol and taxifolin production in YPD medium by buffering media $\mathrm{pH}$ with $40 \mathrm{~g} / \mathrm{L} \mathrm{CaCO}_{3}$ and inhibiting fatty acid synthesis with $1 \mathrm{mg} / \mathrm{L}$ cerulenin. We observed that strains ErioPro and TaxiPro produced $95.5 \mathrm{mg} / \mathrm{L}$ eriodictyol and $79.1 \mathrm{mg} / \mathrm{L}$ taxifolin in $144 \mathrm{~h}$, which were $76.2 \%$ and $64.4 \%$ higher than that without $\mathrm{CaCO}_{3}$ and cerulenin. ErioPro and TaxiPro produced $134.2 \mathrm{mg} / \mathrm{L}$ eriodictyol and $110.5 \mathrm{mg} / \mathrm{L}$ taxifolin at the end of the fermentation process (Fig. 6b). These results indicate that Y. lipolytica is an ideal platform to functionally express plant-derived P450 enzymes. By optimizing the bioprocess, we could substantially improve the titer of naringenin, eriodictyol and taxifolin in metabolically-engineered oleaginous yeast species.

\section{Conclusions}

The heterologous production of hydroxylated flavonoids remains a challenging task; and only limited successful pathway engineering endeavors have been reported to date. Oleaginous yeast is rich in lipid and internal membrane structures, which provides the hydrophobic lipid environment and spatially-organized organelles that are critical for plant P450 enzyme 
315 functionality. In this report, we validated that $Y$. lipolytica is a superior platform for heterologous 316 production of high value flavonoids and hydroxylated flavonoids. By modular construction and 317 characterization of various genes involved in plant flavonoid biosynthesis, we determined that 318 chalcone synthase (CHS), flavanone 3-hydroxylase ( $\mathrm{F} 3 \mathrm{H})$ and cytochrome c $\mathrm{P} 450$ reductase 319 (CrCPR) were the critical steps to engineer flavonoid production in Y. lipolytica. Coupling with 320 the upstream amino acid degradation pathway (tyrosine ammonia lyase from Rhodotorula 321 glutinis), for the first time, we achieved de novo production of naringenin, eriodictyol and 322 taxifolin from glucose in Y. lipolytica. By using a modular cloning platform to assemble multiple 323 genetic constructs, we further determined the optimal gene copy ratio for CHS, F3H and CrCPR 324 to cooperatively improve flavonoids and hydroxylated flavonoids production. We then unleashed 325 the metabolic potential of the yeast host by screening and testing a number of precursor 326 pathways, including the acetyl-CoA synthetase, acetyl-CoA carboxylase and chorismate pathway 327 (the pentafunctional AROM polypeptide ARO1). Coupled with the optimized chalcone synthase 328 module and the hydroxylation module, our engineering strategies synergistically removed 329 pathway bottlenecks and led to a 15.8-fold, 6.9-fold and 8.8-fold improvement in naringenin, 330 eriodictyol and taxifolin production, respectively. Collectively, these findings demonstrate our 331 abilities to harness oleaginous yeast as microbial workhorse to expand nature's biosynthetic 332 potential, which allows us to produce complex natural products from cheap feedstocks.

\section{Materials and methods}

\section{Genes, plasmids, and strains}

335 Genes encoding Rhodotorula $R$. toruloides tyrosine ammonia lyase (RtTAL), Petroselinum 336 crispum (parsley) 4-coumarate-CoA ligase (Pc4CL), Petunia $x$ hybrid chalcone synthase 337 (PhCHS), Medicago sativa chalcone isomerase (MsCHI), Escherichia coli acetyl-CoA 338 synthetase (EcACS), and Corynebacterium glutamicum ATCC 13032 acetyl-CoA carboxylase $339(\mathrm{Cg} A C C)$ were frozen stocks of our laboratory. Genes encoding Solanum lycopersicum 340 4-coumarate-CoA ligase (Sl4CL), Hordeum vulgare chalcone synthase (HvCHS2), Petunia $x$ 341 hybrid chalcone isomerase (PhCHI), Gerbera hybrid flavonoid 3'-hydroxylase (GhF3' $H$ ),

342 Glycine max flavonoid 3'-hydroxylase $(G m F 3 ' H)$, Catharanthus roseus cytochrome P450 343 reductase $(C r C P R)$, and Solanum lycopersicum flavanone 3-hydroxylase (SlF3H) were 344 optimized and synthesized by GenScript (Nanjing, China). Genes encoding Yarrowia lipolytica 345 pentafunctional arom protein (YlARO1), Yarrowia lipolytica cytochrome P450 reductase 346 (YlCPR), Yarrowia lipolytica acetyl-CoA synthetase (YlACS2) were amplified from Yarrowia 347 lipolytica Polf genomic DNA by PCR. Saccharomyces cerevisiae cytochrome P450 reductase 348 (ScCPR1) and Saccharomyces cerevisiae acetyl-CoA synthetase (ScACS2) were amplified from 349 Saccharomyces cerevisiae genomic DNA by PCR. Genes used in this project were listed in 350 Supplementary Table S1.

351 Plasmid pYLXP' was a stock of our laboratory ${ }^{89}$. Plasmid pYLXP'2 was constructed by 
352

353

354

355

356

357

358

359

360

361

362

363

364

365

366

367

368

369

370

371

372

373

374

375

376

377

378

379

380

381

382

383

384

385

386

387

388

389

replacing LEU2 marker with URA3 marker. Both pYLXP' and pYLXP'2 were YaliBrick plasmids and used for flavonoid pathway construction ${ }^{34}$. Escherichia coli (E. coli) NEB $5 \alpha$ was used for plasmid construction, propagation, and maintenance. Yarrowia lipolytica (Y. lipolytica) Po1f (ATCC MYA-2613, MATA ura3-302 leu2-270 xpr2-322 axp2-deltaNU49 XPR2::SUC2) was used as the chassis to construct flavonoid pathways.

To achieve de novo synthesis of eriodictyol and taxifolin, we transformed pYLXP'2-GhF3'H-CrCPR and pYLXP'2-GhF3'H-CrCPR-SIF3H into Po1f/T4SI, resulting in Polf/T4SIHR and Polf/T4SIHRH, respectively. The strain containing 5 copies of PhCHS was named as Po1f/T4S ${ }_{x 5}$ I. We chose to use the plasmids pYLXP'2-HR 2 and pYLXP'2-HR ${ }_{\times 2} \mathrm{H}$, which contain 2 copies of $C r C P R$, to construct eriodictyol and taxifolin pathways. To achieve de novo synthesis of eriodictyol and taxifolin, strains Polf/T4S $\mathrm{S}_{\mathrm{x} 5} \mathrm{IHR}_{\mathrm{x} 2}$ and Po1f/T4 $\mathrm{S}_{\mathrm{x} 5} \mathrm{IHR}_{\mathrm{x} 2} \mathrm{H}$ were constructed by transforming plasmids pYLXP'2-HR $\mathrm{x}_{\mathrm{x} 2}$ and $\mathrm{pYLXP} 2-\mathrm{HR}_{\mathrm{x} 2} \mathrm{H}$ into strain Po1f/T4S ${ }_{x 5}$ I, respectively. We over-expressed Y1ARO1 along with Y1ACS2-Y1ACC1 in strain Po1f/T4S ${ }_{x 5} I$, and name the new strain as Po1f/AT4S ${ }_{x 5} I-Y 1 A C S 2-Y 1 A C C 1$. By introducing Module II into strains Po1f/AT4S ${ }_{x 5} I$ and Po1f/AT4S ${ }_{x 5} I-Y 1 A C S 2-Y 1 A C C 1$, we obtained eriodictyol producing strains Po1f/AT4S $\mathrm{S}_{\mathrm{x} 5} \mathrm{IHR}_{\mathrm{x} 2}$ and Po1f/AT4S $\mathrm{x}_{5} \mathrm{IHR}_{\mathrm{x} 2}-\mathrm{Y} 1 \mathrm{ACS} 2-\mathrm{Y} 1 \mathrm{ACC} 1$ and taxifolin producing strains Po1f/AT4S ${ }_{\mathrm{x} 5} \mathrm{IHR}_{\mathrm{x} 2} \mathrm{H}$ and Po1f/AT4S $\mathrm{x}_{5} \mathrm{IHR}_{\mathrm{x} 2} \mathrm{H}-\mathrm{Y} 1 \mathrm{ACS} 2-\mathrm{Y} 1 \mathrm{ACC} 1$. Strains constructed in this project were listed in Supplementary Table S2.

\section{Pathway construction}

Genes RtTAL, Pc4CL, PhCHS, MsCHI, EcACS, CgACC, YlCPR, YlACS2, ScCPR1, and ScACS2 were amplified using respective primers listed in Supplementary Table S3. The PCR product was assembled with SnaBI digested pYLXP' or pYLXP'2 using Gibson Assembly method. YlARO1 is composed of 2 extrons and 1 intron. The extrons were amplified by using primer pairs ARO1_up F/ARO1_up R and ARO1_down F/ARO1_down R respectively. The resulting PCR products were assembled with SnaBI digested pYLXP' to yield pYLXP'-ARO1, removing the intron sequence. For gene expression, the start codon was removed and a nucleic acid sequence "TAACCGCAG" was added at the upstream of coding gene to complete the intron ${ }^{34}$.

The YaliBrick method was used to assemble the synthetic pathways ${ }^{34}$. pYLXP' derived plasmids were used to assemble the pathways of Module I, while pYLXP'2 derived plasmids were used to assemble the pathways of Module II and ACS and ACC. Generally, the donor plasmids were digested with AvrII/SalI, and the destination plasmids were digested with NheI/SalI. The resulting plasmids containing monocistronic configurations were obtained by T4 ligation. For the assemble of genes containing any of these isocaudomers, other isocaudomers were used. Specifically, the donor plasmid pYLXP'-Y1ARO1 was digested with HpaI/NheI, and the destination plasmid pYLXP'-T4S $\mathrm{S}_{\mathrm{x} 5 \mathrm{I}}$ was digested with $H p a \mathrm{I} / A v r I I$. The resulting plasmid pYLXP'-AT4S ${ }_{\times 5}$ I was obtained by inserting YlARO1 into pYLXP'-T4S ${ }_{x 5}$ I using T4 ligation. The donor plasmid pYLXP'2-HR ${ }_{x 2} \mathrm{H}$ was digested with ClaI/NheI, and the destination plasmid 
390 pYLXP'2-ScACS2-Y1ACC1 was digested with ClaI/AvrII. The resulting plasmid 391 pYLXP'2-HR ${ }_{x 2} \mathrm{H}-\mathrm{ScACS} 2-\mathrm{Y} 1 \mathrm{ACC} 1$ was obtained by inserting genes GhF3'H-CrCPR ${ }_{\mathrm{x} 2}-\mathrm{SIF} 3 \mathrm{H}$ 392 into pYLXP'2-ScACS2-Y1ACC1 using T4 ligation. Plasmids pYLXP'2-Y1ACC1, 393 pYLXP'2-EcACCABCD-EcBirA, and pYLXP'2-Y1BPL1 were frozen stocks of our laboratory

$394 \quad 34$. Plasmids used in this paper were listed in Supplementary Table S4.

\section{Yeast transformation and screening}

The lithium acetate (LiAc) method was used for the transformation. Y. lipolytica was cultured on YPD plate at $30^{\circ} \mathrm{C}$ for $16-22 \mathrm{~h}$. The transformation solution was prepared as follows: $90 \mu \mathrm{L} \mathrm{50 \%} \mathrm{PEG4000,} 5 \mu \mathrm{L} 2 \mathrm{M}$ LiAc, $5 \mu \mathrm{L}$ boiled single strand DNA (salmon sperm, denatured), and 200-500 ng plasmid DNA. The transformation solution was mixed well by vortexing before use. Next, the yeast was transferred to the transformation solution, and mixed well by vortexing for at least 10 seconds. The transformation mixtures were then incubated at $30^{\circ} \mathrm{C}$ for 30-45 min. The transformation mixture was then vortexed for 15 seconds every 10 minutes, followed by an additional $10 \mathrm{~min}$ heat shock at $39^{\circ} \mathrm{C}$ to increase transformation efficiency. For the transformation of pYLXP' and derivative plasmids, the mixture was plated on leucine drop-out complete synthetic media (CSM-Leu). For the transformation of pYLXP'2 and derivative plasmids, the mixture was plated on uracil drop-out complete synthetic media (CSM-Ura). For the transformation of both plasmids, the mixture was plated on leucine and uracil drop-out complete synthetic media (CSM-Leu-Ura). Strains NarPro/ASC, ErioPro, and TaxiPro were constructed in previous work ${ }^{40}$. Strains used in this paper were listed in Strains constructed in this project were listed in Supplementary Table S2.

\section{Cultivation and $\mathbf{p H}$ control}

412 The seed was cultured in regular leucine, or uracil, or leucine and uracil drop-out complete 413 synthetic media (CSM-Leu, or CSM-Ura, or CSM-Leu-Ura) at $30^{\circ} \mathrm{C}$ for 2 days. The seed culture 414 was inoculated to $25 \mathrm{~mL}$ nitrogen-limited media $(\mathrm{C} / \mathrm{N}=80)$ to a final concentration of $2 \%(\mathrm{v} / \mathrm{v})$. 415 The fermentation was carried out in $250 \mathrm{~mL}$ shak flask at $30^{\circ} \mathrm{C} 220 \mathrm{rpm}$. C/N ratio was 416 optimized by two patterns: i) fixing glucose content ( $40 \mathrm{~g} / \mathrm{L})$ and altering $\left(\mathrm{NH}_{4}\right)_{2} \mathrm{SO}_{4}$ content; ii) 417 fixing $\left(\mathrm{NH}_{4}\right)_{2} \mathrm{SO}_{4}$ content $(0.73348 \mathrm{~g} / \mathrm{L})$ and altering glucose content. To analyze the effect of 418 cerulenin, oleic acid, and sodium acetate (NaAc) on flavonoid synthesis, a final concentration of $4195 \mathrm{~g} / \mathrm{L}$ oleic acid or $1 \mathrm{mM} \mathrm{NaAc}$ was added at the starting point, while a final concentration of 1 $420 \mathrm{mg} / \mathrm{L}$ cerulenin was added at $48 \mathrm{~h}$. To buffer the acidity, $20 \mathrm{mM}$ phosphate buffer saline (PBS, $\left.421 \mathrm{Na}_{2} \mathrm{HPO}_{4}-\mathrm{NaH}_{2} \mathrm{PO}_{4}\right)$ or $40 \mathrm{~g} / \mathrm{L} \mathrm{CaCO}_{3}$ was used respectively. In the fed-batch fermentation, the 422 starting glucose concentration was $40 \mathrm{~g} / \mathrm{L}$, and a final concentration of $10 \mathrm{~g} / \mathrm{L}$ glucose was added 423 every $24 \mathrm{~h}$ from $48 \mathrm{~h}$.

\section{Analytical methods}

Samples were taken at $144 \mathrm{~h}$. In the fed-batch fermentation, samples were taken every $24 \mathrm{~h}$. For naringenin, eriodictyol, and taxifolin analysis, samples were diluted in methanol; whole for 
427 glucose analysis, samples were diluted in $\mathrm{H}_{2} \mathrm{O}$. Samples were shaken with glass beads to release

428 the metabolites for analysis. Naringenin, eriodictyol, taxifolin, and glucose were analyzed using 429 Agilent HPLC 1220 as previously described ${ }^{40}$.

\section{Acknowledgements}

431 This work was supported by the Cellular \& Biochem Engineering Program of the National 432 Science Foundation under grant no.1805139, the National Natural Science Foundation of China 433 (31670095, 31770097), the Fundamental Research Funds for the Central Universities 434 (JUSRP51701A), the Distinguished Professor Project of Jiangsu Province. The authors would 435 also like to acknowledge the Department of Chemical, Biochemical and Environmental 436 Engineering at University of Maryland Baltimore County for funding support. YL would like to 437 thank the China Scholarship Council for funding support.

\section{Author contributions}

439 PX and JZ conceived the topic. YL performed genetic engineering and fermentation experiments. $440 \quad$ YL and PX wrote the manuscript. JZ and MK revised the manuscript.

\section{Conflicts of interests}

442 A provisional patent has been filed based on the results of this study. 


\section{References}

445 1. Atanasov, A. G.; Waltenberger, B.; Pferschy-Wenzig, E.-M.; Linder, T.; Wawrosch, C.; Uhrin, P.; 446 Temml, V.; Wang, L.; Schwaiger, S.; Heiss, E. H.; Rollinger, J. M.; Schuster, D.; Breuss, J. M.; Bochkov, V.; Mihovilovic, M. D.; Kopp, B.; Bauer, R.; Dirsch, V. M.; Stuppner, H., Discovery and resupply of pharmacologically active plant-derived natural products: A review. Biotechnology Advances 2015, $33(8), 1582-1614$.

2. Chemler, J.; Koffas, M., Metabolic engineering for plant natural product biosynthesis in microbes. Current Opinion in Biotechnology 2008, 597-605.

3. Chemler, J.; Yan, Y.; Koffas, M., Biosynthesis of isoprenoids, polyunsaturated fatty acids and flavonoids in Saccharomyces cerevisiae. Microbial Cell Factories 2006, -.

4. Keasling, J., Manufacturing Molecules Through Metabolic Engineering. Science 2010, 330 (6009), $1355-1358$.

5. Lee, J. W.; Kim, T. Y.; Jang, Y. S.; Choi, S.; Lee, S. Y., Systems metabolic engineering for chemicals and materials. Trends Biotechnol 2011, 29 (8), 370-8.

6. Leonard, E.; Nielsen, D.; Solomon, K.; Prather, K., Engineering microbes with synthetic biology frameworks. Trends in Biotechnology 2008, 26 (12), 674-681.

7. Medema, M.; Breitling, R.; Bovenberg, R.; Takano, E., Exploiting plug-and-play synthetic biology for drug discovery and production in microorganisms. Nature Reviews Microbiology 2011, 131-137.

8. Smanski, M. J.; Zhou, H.; Claesen, J.; Shen, B.; Fischbach, M. A.; Voigt, C. A., Synthetic biology to access and expand nature's chemical diversity. Nat Rev Micro 2016, 14 (3), 135-149.

9. Xu, P.; Bhan, N.; Koffas, M. A. G., Engineering plant metabolism into microbes: from systems biology to synthetic biology. Current Opinion in Biotechnology 2013, 24 (2), 291-299.

10. Palmer, C. M.; Alper, H. S., Expanding the chemical palette of industrial microbes: metabolic engineering for type III pks-derived polyketides. Biotechnol J. 2018, 14.

11. Yuan, S.-F.; Alper, H. S., Metabolic engineering of microbial cell factories for production of nutraceuticals. Microbial Cell Factories 2019, 18 (1), 46.

12. Xiu, Y.; Jang, S.; Jones, J. A.; Zill Nicholas, A.; Linhardt Robert, J.; Yuan, Q.; Jung Gyoo, Y.; Koffas Mattheos, A. G., Naringenin-responsive riboswitch-based fluorescent biosensor module for Escherichia coli co-cultures. Biotechnology and Bioengineering 2017, 114 (10), 2235-2244.

13. Zha, J.; Koffas, M. A. G., Anthocyanin Production in Engineered Microorganisms. In Biotechnology of Natural Products, Schwab, W.; Lange, B. M.; Wüst, M., Eds. Springer International Publishing: Cham, 2018; pp 81-97.

14. Zang, Y.; Zha, J.; Wu, X.; Zheng, Z.; Ouyang, J.; Koffas, M. A. G., In vitro Naringenin Biosynthesis from p-Coumaric Acid Using Recombinant Enzymes. Journal of Agricultural and Food Chemistry 2019. 
15. Blazeck, J.; Hill, A.; Liu, L.; Knight, R.; Miller, J.; Pan, A.; Otoupal, P.; Alper, H. S., Harnessing Yarrowia lipolytica lipogenesis to create a platform for lipid and biofuel production. Nat Commun 2014, 5, 3131.

16. Xu, P.; Qiao, K.; Stephanopoulos, G., Engineering oxidative stress defense pathways to build a robust lipid production platform in Yarrowia lipolytica. Biotechnol Bioeng 2017, 114 (7), 1521-1530.

17. Bailey, R.; Madden, K. T.; Trueheart, J. Production of carotenoids in oleaginous yeast and fungi. US 7,851,199, 2010.

18. Sharpe, P. L.; Rick, W. Y.; Zhu, Q. Q. Carotenoid production in a recombinant oleaginous yeast. US 8,846,374, 2014.

19. Qiao, K. J.; Wasylenko, T. M.; Zhou, K.; Xu, P.; Stephanopoulos, G., Lipid production in Yarrowia lipolytica is maximized by engineering cytosolic redox metabolism. Nat. Biotechnol. 2017, 35 (2), 173-177.

20. Markham, K. A.; Palmer, C. M.; Chwatko, M.; Wagner, J. M.; Murray, C.; Vazquez, S.; Swaminathan, A.; Chakravarty, I.; Lynd, N. A.; Alper, H. S., Rewiring Yarrowia lipolytica toward triacetic acid lactone for materials generation. Proc. Natl. Acad. Sci. U. S. A. 2018, 115 (9), 2096-2101.

21. Larroude, M.; Celinska, E.; Back, A.; Thomas, S.; Nicaud, J. M.; Ledesma-Amaro, R., A synthetic biology approach to transform Yarrowia lipolytica into a competitive biotechnological producer of $\beta$-carotene. Biotechnol. Bioeng. 2018, 115 (2), 464-472.

22. Groenewald, M.; Boekhout, T.; Neuvéglise, C.; Gaillardin, C.; van Dijck, P. W. M.; Wyss, M., Yarrowia lipolytica: Safety assessment of an oleaginous yeast with a great industrial potential. Critical Reviews in Microbiology 2014, 40 (3), 187-206.

23. Cui, Z.; Gao, C.; Li, J.; Hou, J.; Lin, C. S. K.; Qi, Q., Engineering of unconventional yeast Yarrowia lipolytica for efficient succinic acid production from glycerol at low pH. Metabolic Engineering 2017, 42, 126-133.

24. Abghari, A.; Chen, S., Yarrowia lipolytica as an oleaginous cell factory platform for the production of fatty acid-based biofuel and bioproducts. Frontiers in Energy Research 2014, 2.

25. Ledesma-Amaro, R.; Lazar, Z.; Rakicka, M.; Guo, Z.; Fouchard, F.; Coq, A.-M. C.-L.; Nicaud, J.-M., Metabolic engineering of Yarrowia lipolytica to produce chemicals and fuels from xylose. Metabolic Engineering 2016, 38, 115-124.

26. Li, H.; Alper, H. S., Enabling xylose utilization in Yarrowia lipolytica for lipid production. Biotechnology Journal 2016, 11 (9), 1230-1240.

27. Rodriguez, G. M.; Hussain, M. S.; Gambill, L.; Gao, D.; Yaguchi, A.; Blenner, M., Engineering xylose utilization in Yarrowia lipolytica by understanding its cryptic xylose pathway. Biotechnology for Biofuels 2016, $9(1), 149$.

28. Bordes, F.; Fudalej, F.; Dossat, V.; Nicaud, J. M.; Marty, A., A new recombinant protein expression system for high-throughput screening in the yeast Yarrowia lipolytica. J Microbiol Methods 2007, 70 (3), 
29. Juretzek, T.; Le Dall, M.; Mauersberger, S.; Gaillardin, C.; Barth, G.; Nicaud, J., Vectors for gene expression and amplification in the yeast Yarrowia lipolytica. Yeast 2001, 18 (2), 97-113.

30. Nicaud, J. M.; Madzak, C.; Broek, P.; Gysler, C.; Duboc, P.; Niederberger, P.; Gaillardin, C., Protein expression and secretion in the yeast Yarrowia lipolytica. FEMS Yeast Res 2002, 2.

31. Blazeck, J.; Liu, L.; Redden, H.; Alper, H., Tuning gene expression in Yarrowia lipolytica by a hybrid promoter approach. Appl Environ Microbiol 2011, 77.

32. Blazeck, J.; Reed, B.; Garg, R.; Gerstner, R.; Pan, A.; Agarwala, V.; Alper, H. S., Generalizing a hybrid synthetic promoter approach in Yarrowia lipolytica. Appl Microbiol Biotechnol 2013, 97.

33. Liu, H.; Marsafari, M.; Deng, L.; Xu, P., Understanding lipogenesis by dynamically profiling transcriptional activity of lipogenic promoters in Yarrowia lipolytica. Applied Microbiology and Biotechnology 2019, 103 (7), 3167-3179.

34. Wong, L.; Engel, J.; Jin, E.; Holdridge, B.; Xu, P., YaliBricks, a versatile genetic toolkit for streamlined and rapid pathway engineering in Yarrowia lipolytica. Metab. Eng. Commun. 2017, 5, 68-77.

35. Wong, L.; Holdridge, B.; Engel, J.; Xu, P., Genetic Tools for Streamlined and Accelerated Pathway Engineering in Yarrowia lipolytica. In Microbial Metabolic Engineering: Methods and Protocols, Santos, C. N. S.; Ajikumar, P. K., Eds. Springer New York: New York, NY, 2019; pp 155-177.

36. Celińska, E.; Ledesma-Amaro, R.; Larroude, M.; Rossignol, T.; Pauthenier, C.; Nicaud, J.-M., Golden Gate Assembly system dedicated to complex pathway manipulation in Yarrowia lipolytica. Microbial Biotechnology 2017, 10 (2), 450-455.

37. Wagner, J. M.; Williams, E. V.; Alper, H. S., Developing a piggyBac Transposon System and Compatible Selection Markers for Insertional Mutagenesis and Genome Engineering in Yarrowia lipolytica. Biotechnology Journal 2018, 13 (5), 1800022.

38. Schwartz, C. M.; Hussain, M. S.; Blenner, M.; Wheeldon, I., Synthetic RNA polymerase III promoters facilitate high-efficiency CRISPR-Cas9-mediated genome editing in Yarrowialipolytica. ACS Synth Biol 2016, 5 .

39. Morse, N. J.; Wagner, J. M.; Reed, K. B.; Gopal, M. R.; Lauffer, L. H.; Alper, H. S., T7 Polymerase Expression of Guide RNAs in vivo Allows Exportable CRISPR-Cas9 Editing in Multiple Yeast Hosts. ACS Synthetic Biology 2018, 7 (4), 1075-1084.

40. Lv, Y.; Edwards, H.; Zhou, J.; Xu, P., Combining 26s rDNA and the Cre-loxP system for iterative gene integration and efficient marker curation in Yarrowia lipolytica. ACS Synth. Biol. 2019.

41. Dixon, R. A.; Pasinetti, G. M., Flavonoids and Isoflavonoids: From Plant Biology to Agriculture and Neuroscience. Plant Physiology 2010, 154 (2), 453. 
546

547

548

549

550

551

552

553

554

555

556

557

558

559

560

561

562

563

564

565

566

567

568

569

570

571

572

573

574

575

576

577

578

579

flavonoids: Pathway engineering, directed evolution and systems/synthetic biology. Biotechnology Advances 2016, 34 (5), 634-662.

43. Benavente-Garcia, O.; Castillo, J.; Alcaraz, M.; Vicente, V.; Del Rio, J.; Ortuno, A., Beneficial action of Citrus flavonoids on multiple cancer-related biological pathways. Current Cancer Drug Targets 2007, 795-809.

44. Benavente-García, O.; Castillo, J., Update on uses and properties of citrus flavonoids: new findings in anticancer, cardiovascular, and anti-inflammatory activity. J Agric Food Chem 2008, 56 (15), 6185-205.

45. Butelli, E.; Titta, L.; Giorgio, M.; Mock, H.-P.; Matros, A.; Peterek, S.; Schijlen, E. G. W. M.; Hall, R. D.; Bovy, A. G.; Luo, J.; Martin, C., Enrichment of tomato fruit with health-promoting anthocyanins by expression of select transcription factors. Nature Biotechnology 2008, 26, 1301.

46. Xu, P.; Ranganathan, S.; Fowler, Z.; Maranas, C.; Koffas, M., Genome-scale metabolic network modeling results in minimal interventions that cooperatively force carbon flux towards malonyl-CoA. Metabolic Engineering 2011, 13 (5), 578-587.

47. Santos, C. N. S.; Koffas, M.; Stephanopoulos, G., Optimization of a heterologous pathway for the production of flavonoids from glucose. Metabolic Engineering 2011, 13 (4), 392-400.

48. Leonard, E.; Yan, Y.; Lim, K.; Koffas, M., Investigation of two distinct flavone synthases for plant-specific flavone biosynthesis in Saccharomyces cerevisiae. Applied and Environmental Microbiology 2005, 8241-8248.

49. Leonard, E.; Lim, K.; Saw, P.; Koffas, M., Engineering central metabolic pathways for high-level flavonoid production in Escherichia coli. Applied and Environmental Microbiology 2007, 3877-3886.

50. Lim, C.; Fowler, Z.; Hueller, T.; Schaffer, S.; Koffas, M., High-Yield Resveratrol Production in Engineered Escherichia coli. Applied and Environmental Microbiology 2011, 3451-3460.

51. Li, M.; Kildegaard, K. R.; Chen, Y.; Rodriguez, A.; Borodina, I.; Nielsen, J., De novo production of resveratrol from glucose or ethanol by engineered Saccharomyces cerevisiae. Metab Eng 2015, 32.

52. Li, M.; Schneider, K.; Kristensen, M.; Borodina, I.; Nielsen, J., Engineering yeast for high-level production of stilbenoid antioxidants. Sci Rep 2016, 6 .

53. Wu, J. J.; Liu, P. R.; Fan, Y. M.; Bao, H.; Du, G. C.; Zhou, J. W.; Chen, J., Multivariate modular metabolic engineering of Escherichia coli to produce resveratrol from L-tyrosine. J. Biotechnol. 2013, 167 (4), 404-411.

54. Bhan, N.; Xu, P.; Khalidi, O.; Koffas, M. A. G., Redirecting carbon flux into malonyl-CoA to improve resveratrol titers: Proof of concept for genetic interventions predicted by OptForce computational framework. Chemical Engineering Science 2013, 103, 109-114.

55. Wu, J.; Du, G.; Zhou, J.; Chen, J., Metabolic engineering of Escherichia coli for (2S)-pinocembrin production from glucose by a modular metabolic strategy. Metabolic Engineering 2013, 16, 48-55.

56. Yan, Y.; Chemler, J.; Huang, L.; Martens, S.; Koffas, M., Metabolic engineering of anthocyanin 
57. Yan, Y.; Li, Z.; Koffas, M., High-yield anthocyanin biosynthesis in engineered Escherichia coli. Biotechnology and Bioengineering 2008, 126-140.

58. Lim, C. G.; Wong, L.; Bhan, N.; Dvora, H.; Xu, P.; Venkiteswaran, S.; Koffas, M. A., Development of a Recombinant Escherichia coli strain for Overproduction of Plant Pigment, Anthocyanin. Appl Environ Microbiol 2015.

59. Rodriguez, A.; Strucko, T.; Stahlhut, S. G.; Kristensen, M.; Svenssen, D. K.; Forster, J.; Nielsen, J.; Borodina, I., Metabolic engineering of yeast for fermentative production of flavonoids. Bioresour Technol 2017, 245 .

60. Lv, Y.; Xu, S.; Lyu, Y.; Zhou, S.; Du, G.; Chen, J.; Zhou, J., Engineering enzymatic cascades for the efficient biotransformation of eugenol and taxifolin to silybin and isosilybin. Green Chemistry 2019, 21 (7), 1660-1667.

61. Li, J.; Tian, C.; Xia, Y.; Mutanda, I.; Wang, K.; Wang, Y., Production of plant-specific flavones baicalein and scutellarein in an engineered E. coli from available phenylalanine and tyrosine. Metabolic Engineering 2019, 52, 124-133.

62. Winkel-Shirley, B., Flavonoid Biosynthesis. A Colorful Model for Genetics, Biochemistry, Cell Biology, and Biotechnology. Plant Physiology 2001, 126 (2), 485.

63. Lin, Y.; Yan, Y., Biotechnological production of plant-specific hydroxylated phenylpropanoids. Biotechnology and Bioengineering 2014, 111 (9), 1895-1899.

64. Leonard, E.; Koffas, M., Engineering of artificial plant cytochrome p450 enzymes for synthesis of isoflavones by Escherichia coli. Applied and Environmental Microbiology 2007, 7246-7251.

65. Negretti, S.; Narayan, A. R. H.; Chiou, K. C.; Kells, P. M.; Stachowski, J. L.; Hansen, D. A.; Podust, L. M.; Montgomery, J.; Sherman, D. H., Directing Group-Controlled Regioselectivity in an Enzymatic C-H Bond Oxygenation. Journal of the American Chemical Society 2014, 136 (13), 4901-4904.

66. Eichmann, T. O.; Kumari, M.; Haas, J. T.; Farese, R. V.; Zimmermann, R.; Lass, A.; Zechner, R., Studies on the Substrate and Stereo/Regioselectivity of Adipose Triglyceride Lipase, Hormone-sensitive Lipase, and Diacylglycerol-O-acyltransferases. Journal of Biological Chemistry 2012, 287 (49), 41446-41457. Gelboin, H. V.; Waxman, D. J.; Gonzalez, F. J., Sequence requirements for cytochrome P-450IIB1 catalytic activity. Alteration of the stereospecificity and regioselectivity of steroid hydroxylation by a simultaneous change of two hydrophobic amino acid residues to phenylalanine. Journal of Biological Chemistry 1989, 264 (35), 21327-21333.

613

68. Kotopka, B. J.; Li, Y.; Smolke, C. D., Synthetic biology strategies toward heterologous phytochemical

614 69. Fowler, Z. L.; Gikandi, W. W.; Koffas, M. A., Increased malonyl coenzyme A biosynthesis by tuning the 
615 Escherichia coli metabolic network and its application to flavanone production. Appl. Environ. Microbiol.

$616 \quad 2009,75(18), 5831-9$.

617 70. Johnson, A. O.; Gonzalez-Villanueva, M.; Wong, L.; Steinbüchel, A.; Tee, K. L.; Xu, P.; Wong, T. S., 618 Design and application of genetically-encoded malonyl-CoA biosensors for metabolic engineering of microbial 619 cell factories. Metabolic Engineering 2017, 44, 253-264.

71. Zha, W. J.; Rubin-Pitel, S. B.; Shao, Z. Y.; Zhao, H. M., Improving cellular malonyl-CoA level in Escherichia coli via metabolic engineering. Metab. Eng. 2009, 11 (3), 192-198.

622

72. Nthangeni, M. B.; Urban, P.; Pompon, D.; Smit, M. S.; Nicaud, J. M., The use of Yarrowia lipolytica for the expression of human cytochrome P450CYP1A1. Yeast 2004, 21 (7), 583-592.

73. Zhang, H.; Stephanopoulos, G., Engineering E. coli for caffeic acid biosynthesis from renewable sugars. Applied Microbiology and Biotechnology 2013, 97 (8), 3333-3341.

74. Ferrara, M. A.; Almeida, D. S.; Siani, A. C.; Lucchetti, L.; Lacerda, P. S. B.; Freitas, A.; Tappin, M. R. R.; Bon, E. P. S., Bioconversion of R-(+)-limonene to perillic acid by the yeast Yarrowia lipolytica. Brazilian Journal of Microbiology 2013, 44, 1075-1080.

75. van Rensburg, E.; Moleleki, N.; van der Walt, J. P.; Botes, P. J.; van Dyk, M. S., Biotransformation of $(+)$ limonene and (-)piperitone by yeasts and yeast-like fungi. Biotechnology Letters 1997, 19 (8), 779-782.

76. Wong, L.; Engel, J.; Jin, E.; Holdridge, B.; Xu, P., YaliBricks, a versatile genetic toolkit for streamlined and rapid pathway engineering in Yarrowia lipolytica. Metabolic Engineering Communications 2017, 5 (Supplement C), 68-77.

77. Rittle, J.; Green, M. T., Cytochrome P450 compound I: capture, characterization, and C-H bond activation kinetics. Science 2010, 330 (6006), 933-7.

78. Parage, C.; Foureau, E.; Kellner, F.; Burlat, V.; Mahroug, S.; Lanoue, A.; Dugé de Bernonville, T.; Londono, M. A.; Carqueijeiro, I.; Oudin, A.; Besseau, S.; Papon, N.; Glévarec, G.; Atehortùa, L.; Giglioli-Guivarc'h, N.; St-Pierre, B.; Clastre, M.; O’Connor, S. E.; Courdavault, V., Class II Cytochrome P450 Reductase Governs the Biosynthesis of Alkaloids. Plant Physiology 2016, 172 (3), 1563.

79. Leonard, E.; Yan, Y.; Lim, K. H.; Koffas, M. A. G., Investigation of Two Distinct Flavone Synthases for Plant-Specific Flavone Biosynthesis in $<\mathrm{em}>$ Saccharomyces cerevisiae $</ \mathrm{em}>$. Applied and Environmental Microbiology 2005, 71 (12), 8241.

80. Braun, A.; Geier, M.; Bühler, B.; Schmid, A.; Mauersberger, S.; Glieder, A., Steroid biotransformations in biphasic systems with Yarrowia lipolytica expressing human liver cytochrome P450 genes. Microbial Cell Factories 2012, 11 (1), 106.

81. Nthangeni, M. B.; Urban, P.; Pompon, D.; Smit, M. S.; Nicaud, J. M., The use of Yarrowia lipolytica for the expression of human cytochrome P450 CYP1A1. Yeast 2004, 21 (7), 583-92.

82. Xu, P.; Gu, Q.; Wang, W.; Wong, L.; Bower, A. G. W.; Collins, C. H.; Koffas, M. A. G., Modular optimization of multi-gene pathways for fatty acids production in E. coli. Nature Communications 2013, 4, 
1409.

651

652

653

654

655

656

657

658

659

660

661

662

663

664

665

666

667

668

669

670

671

672

673

674

675

676

677

678

679

680

681

682

683

684

685

83. Leonard, E.; $\quad$ Ajikumar, P.; $\quad$ Thayer, K.; Xiao, W.; Mo, J.; $\quad$ Tidor, B.; Stephanopoulos, G.; Prather, K., Combining metabolic and protein engineering of a terpenoid biosynthetic pathway for overproduction and selectivity control. Proc Natl Acad Sci U S AProc Natl Acad Sci U S A 2010, 107, 13654-13659.

84. Zelcbuch, L.; Antonovsky, N.; Bar-Even, A.; Levin-Karp, A.; Barenholz, U.; Dayagi, M.; Liebermeister, W.; Flamholz, A.; Noor, E.; Amram, S.; Brandis, A.; Bareia, T.; Yofe, I.; Jubran, H.; Milo, R., Spanning high-dimensional expression space using ribosome-binding site combinatorics. Nucleic Acids Research 2013, 41 (9), e98-e98.

85. He, L.; Xiu, Y.; Jones, J. A.; Baidoo, E. E. K.; Keasling, J. D.; Tang, Y. J.; Koffas, M. A. G., Deciphering flux adjustments of engineered E. coli cells during fermentation with changing growth conditions. Metabolic Engineering 2017, 39, 247-256.

86. Munro, A. W.; Girvan, H. M.; Mason, A. E.; Dunford, A. J.; McLean, K. J., What makes a P450 tick? Trends Biochem. Sci. 2013, 38 (3), 140-150.

87. Duncan, K.; Edwards, R. M.; Coggins, J. R., The pentafunctional arom enzyme of Saccharomyces cerevisiae is a mosaic of monofunctional domains. Biochem $J$ 1987, 246 (2), 375-386.

88. Dujon, B.; Sherman, D.; Fischer, G.; Durrens, P.; Casaregola, S.; Lafontaine, I.; De Montigny, J.; Marck, C.; Neuveglise, C.; Talla, E.; Goffard, N.; Frangeul, L.; Aigle, M.; Anthouard, V.; Babour, A.; Barbe, V.; Barnay, S.; Blanchin, S.; Beckerich, J. M.; Beyne, E.; Bleykasten, C.; Boisrame, A.; Boyer, J.; Cattolico, L.; Confanioleri, F.; De Daruvar, A.; Despons, L.; Fabre, E.; Fairhead, C.; Ferry-Dumazet, H.; Groppi, A.; Hantraye, F.; Hennequin, C.; Jauniaux, N.; Joyet, P.; Kachouri, R.; Kerrest, A.; Koszul, R.; Lemaire, M.; Lesur, I.; Ma, L.; Muller, H.; Nicaud, J. M.; Nikolski, M.; Oztas, S.; Ozier-Kalogeropoulos, O.; Pellenz, S.; Potier, S.; Richard, G. F.; Straub, M. L.; Suleau, A.; Swennen, D.; Tekaia, F.; Wesolowski-Louvel, M.; Westhof, E.; Wirth, B.; Zeniou-Meyer, M.; Zivanovic, I.; Bolotin-Fukuhara, M.; Thierry, A.; Bouchier, C.; Caudron, B.; Scarpelli, C.; Gaillardin, C.; Weissenbach, J.; Wincker, P.; Souciet, J. L., Genome evolution in yeasts. Nature 2004, 430 (6995), 35-44.

89. Xu, P.; Qiao, K. J.; Ahn, W. S.; Stephanopoulos, G., Engineering Yarrowia lipolytica as a platform for synthesis of drop-in transportation fuels and oleochemicals. Proc. Natl. Acad. Sci. U. S. A. 2016, 113 (39), 10848-10853.

90. Beopoulos, A.; Nicaud, J. M.; Gaillardin, C., An overview of lipid metabolism in yeasts and its impact on biotechnological processes. Appl. Microbiol. Biotechnol. 2011, 90 (4), 1193-206.

91. Zhu, S.; Wu, J.; Du, G.; Zhou, J.; Chen, J., Efficient synthesis of eriodictyol from L-tyrosine in Escherichia coli. Appl. Environ. Microbiol. 2014, 80 (10), 3072-3080.

92. Davis, M. S.; Solbiati, J.; Cronan, J. E., Jr., Overproduction of acetyl-CoA carboxylase activity increases the rate of fatty acid biosynthesis in Escherichia coli. J Biol Chem 2000, 275 (37), 28593-8.

93. Hoja, U.; Wellein, C.; Greiner, E.; Schweizer, E., Pleiotropic phenotype of 
686

687

688

689

690

691

692

693

694

695

696

697

698

699

700

701

702

703
acetyl-CoA-carboxylase-defective yeast cells - Viability of a BPL1-amber mutation depending on its readthrough by normal tRNA ${ }^{\mathrm{GlnCAG}}$. Eur. J. Biochem. 1998, 254 (3), 520-6.

94. Chakravartty, V.; Cronan, J. E., Altered regulation of Escherichia coli biotin biosynthesis in BirA superrepressor mutant strains. J. Bacteriol. 2012, 194 (5), 1113-1126.

95. Cronan, J. E.; Wallace, J. C., The gene encoding the biotin-apoprotein ligase of Saccharomyces cerevisiae. Fems Microbiol Lett 1995, 130 (2-3), 221-229.

96. Braunwald, T.; Schwemmlein, L.; Graeff-Honninger, S.; French, W. T.; Hernandez, R.; Holmes, W. E.; Claupein, W., Effect of different $\mathrm{C} / \mathrm{N}$ ratios on carotenoid and lipid production by Rhodotorula glutinis. Appl. Microbiol. Biotechnol. 2013, 97 (14), 6581-8.

97. Ratledge, C.; Wynn, J. P., The biochemistry and molecular biology of lipid accumulation in oleaginous microorganisms. Adv Appl Microbiol 2002, 51.

98. Lv, Y.; Edwards, H.; Zhou, J.; Xu, P., Combining 26s rDNA and the Cre-loxP System for Iterative Gene Integration and Efficient Marker Curation in Yarrowia lipolytica. ACS Synthetic Biology 2019, 8 (3), 568-576.

99. Xu, P.; Wang, W.; Li, L.; Bhan, N.; Zhang, F.; Koffas, M. A. G., Design and Kinetic Analysis of a Hybrid Promoter-Regulator System for Malonyl-CoA Sensing in Escherichia coli. ACS Chemical Biology 2014, 9 (2), 451-458. 


\section{Tables}

705

\begin{tabular}{|l|c|c|c|}
\hline \multicolumn{3}{|c|}{ Table 1. Comparison of E. coli, S. cerevisiae and . lipolytica as chassis to } \\
produce plant natural products \\
\hline Expression platform & E. coli & S. cerevisiae & Y. lipolytica \\
\hline Genetic tools & +++++ & +++++ & +++ \\
\hline Genome annotation & ++++ & ++++ & ++++ \\
\hline Acetyl-CoA/Malonyl-CoA/HMG-CoA flux & ++ & +++ & +++++ \\
\hline P450 expression & + & +++ & +++++ \\
\hline Substrate flexibility & ++++ & ++ & ++++ \\
\hline Acid tolerance & +++ & ++ & +++++ \\
\hline FDA safety & + & +++++ & +++++ \\
\hline Hydrophobic lipid body environment & + & ++ & +++++ \\
\hline
\end{tabular}

706 


\section{Figure and legends}

708

709

710

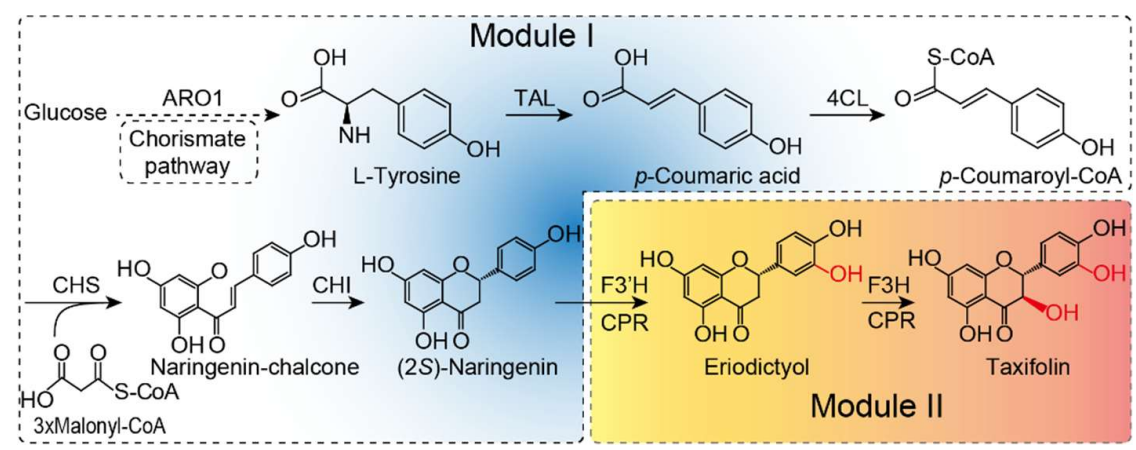

711 Fig. 1. Modular strategy to optimize naringenin, eriodictyol and taxifolin pathways. Based on

712 the reaction cascades, flavonoid pathway was partitioned into 2 modules, naringenin synthetic 713 module (Module I) and hydroxylation module (Module II). Module I contains chorismate 714 pathway and malonyl-CoA utilizing step, and Module II contains flavanone 3-hydroxyase and 715 cytochrome c P450 reductases. 
717

718

719

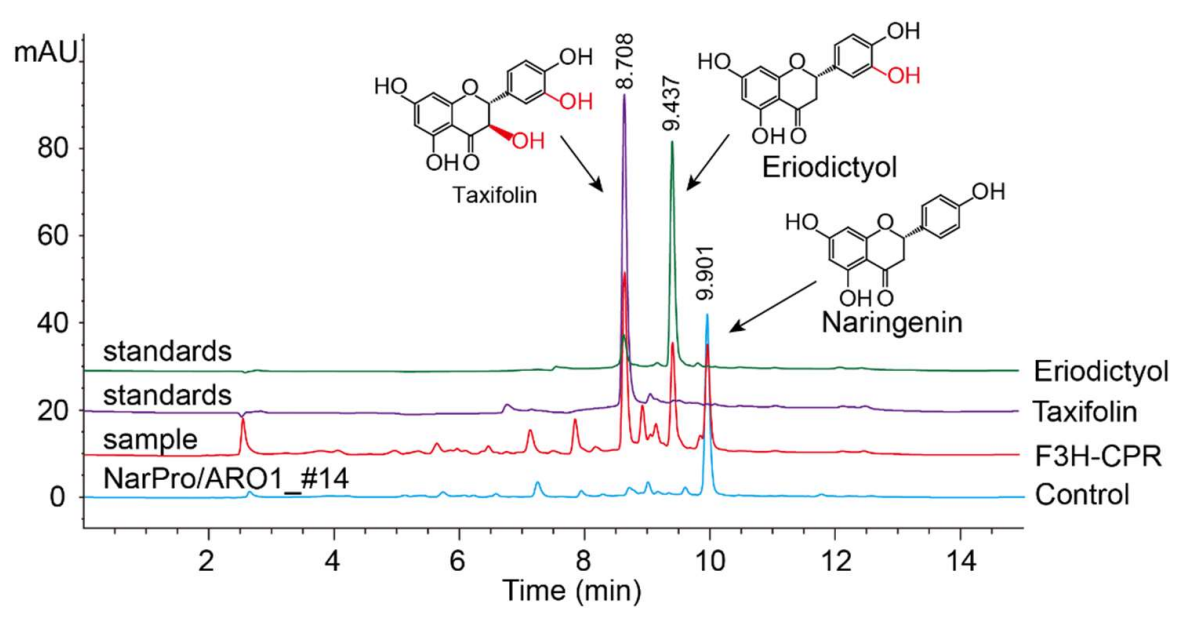

720 Fig. 2. HPLC profile of naringenin, eriodictyol and taxifolin. Two hydroxylated flavonoid 721 standards (taxifolin, purple and eriodictyol, green) were injected to HPLC. One 722 naringenin-producing sample (blue) and one taxifolin-producing sample (red) are shown in the 723 chromatogram. 

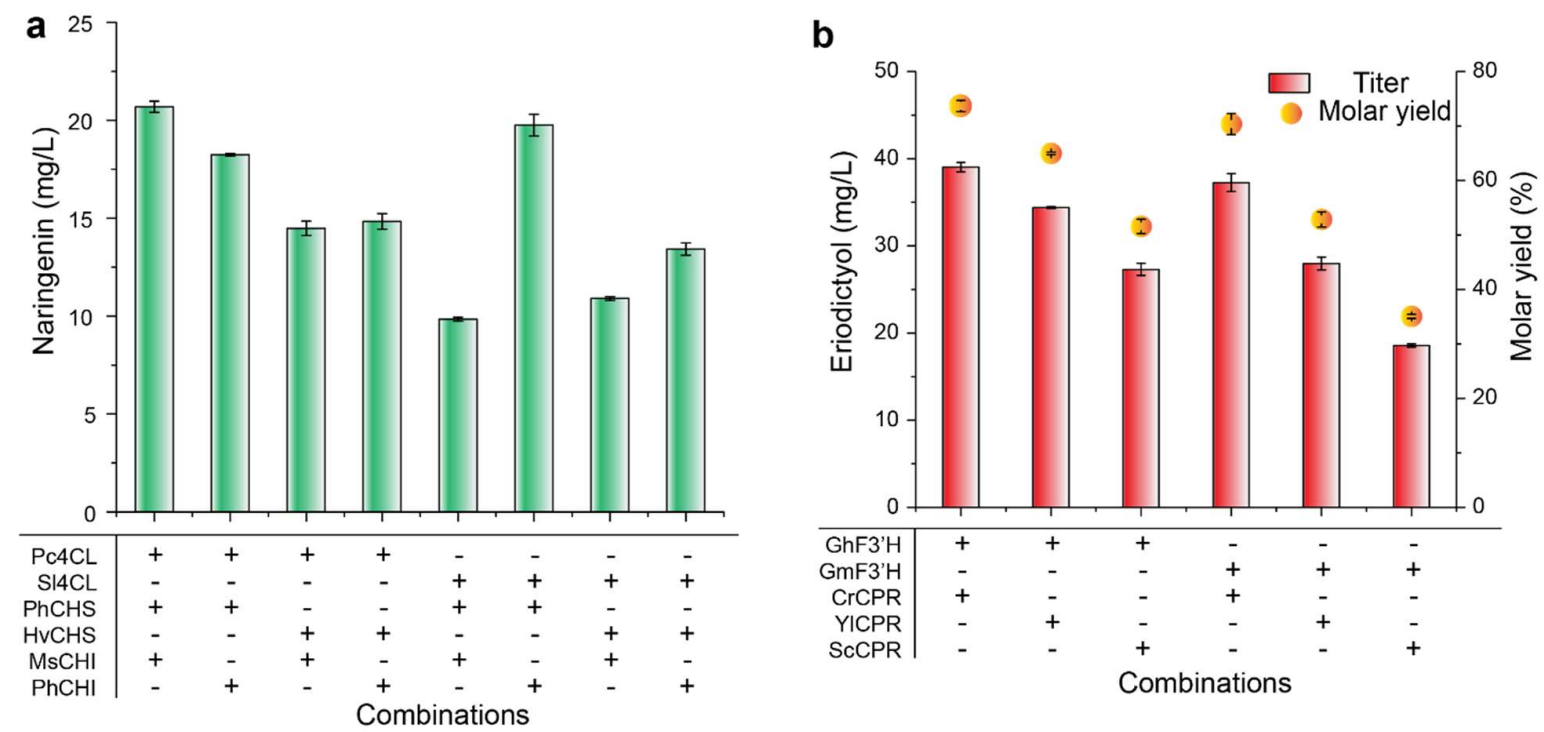

Fig. 3. Screening of gene combinations for improving flavonoid production. (a) Screening of $4 \mathrm{CH}, \mathrm{CHS}$ and $\mathrm{CHI}$ genes from different pants for naringenin production. (b) Screening of $F 3$ ' $H$ and $C P R$ genes from different organisms for eriodictyol production. Plant name and gene sources can be found in supplementary table S1. 

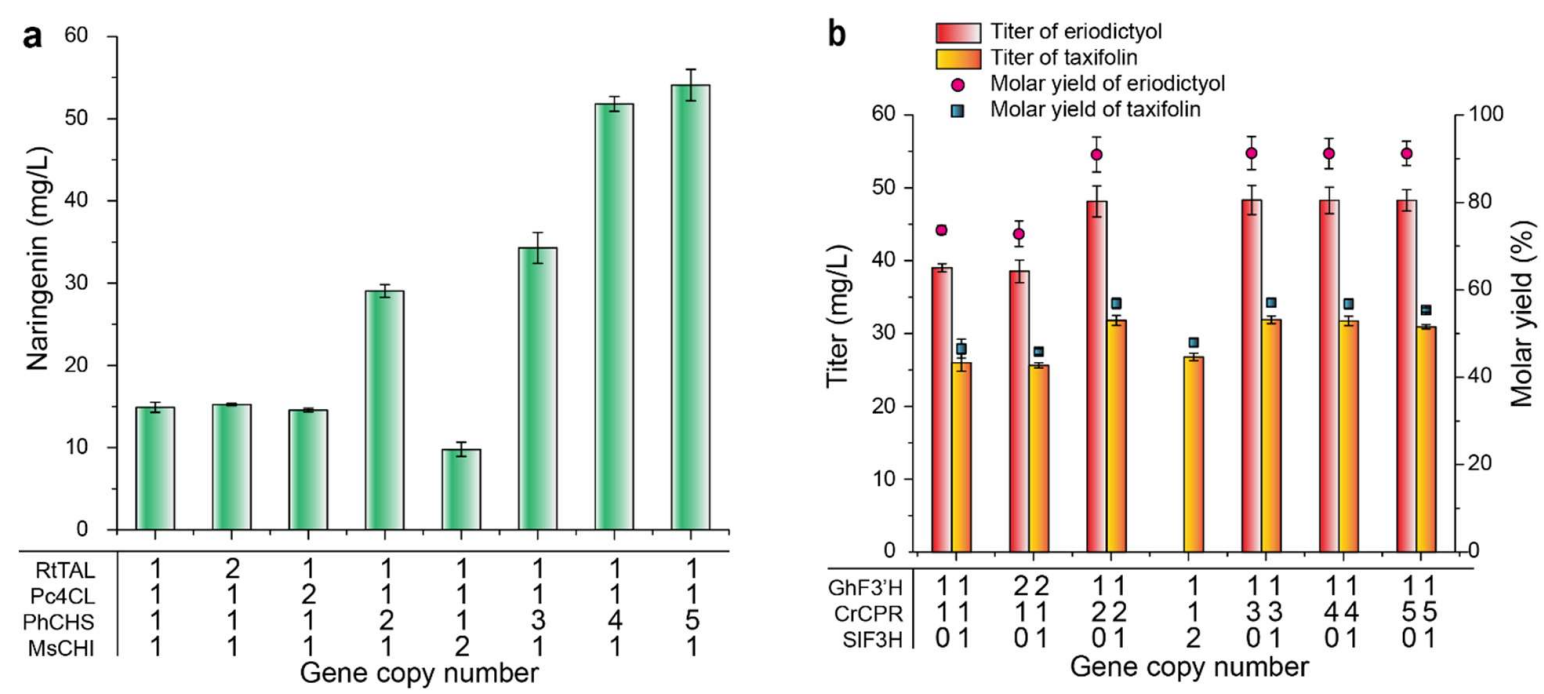

750 Fig. 4. Overcoming rate-limiting steps by tuning gene copy numbers. Rate-limiting steps were determined by gradually increasing gene copy number of each step. Numbers refer to gene copy numbers. (a) Rate-limiting step analysis and optimization of module I to improve naringenin production. (b) Rate-limiting step analysis and optimization of module II to improve eriodictyol and taxifolin production. The molar yield was calculated using $50 \mathrm{mg} / \mathrm{L}$ naringenin as feeding substrate. The number 0 refers to the module that does not contain the respective gene. 

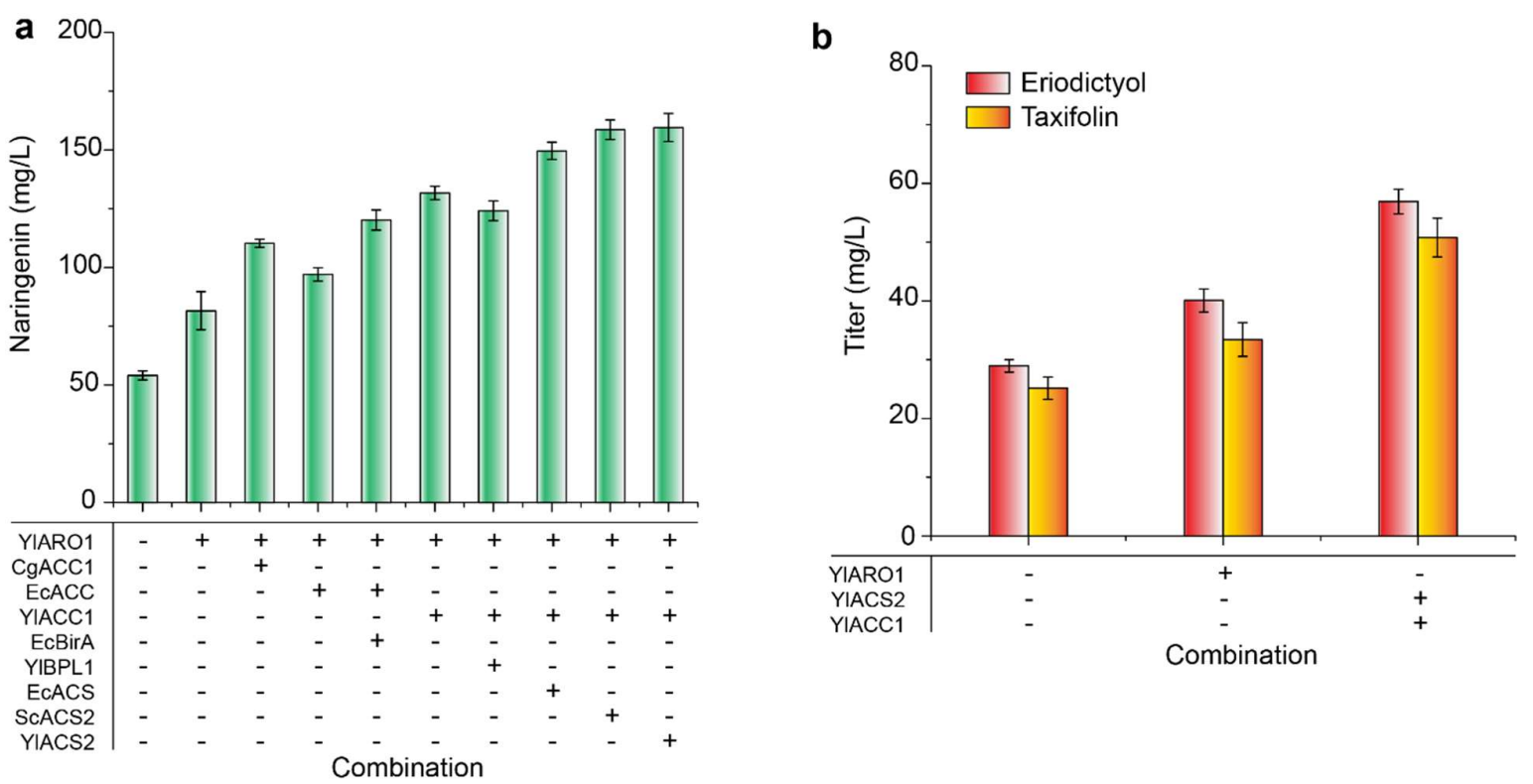

Fig. 5. Improving naringenin, eriodictyol, and taxifolin production by enhancing precursor synthesis. (a) Identification of possible rate-limiting steps by overexpression of chorismate pathway (ARO1), malonyl-CoA pathway (ACC) and acetyl-CoA pathway (ACS). The related genes were overexpressed in strains Po1f/T4S $\mathrm{S}_{\mathrm{x} 5} \mathrm{I}$. (b) Effects of improving malonyl-CoA and chorismate synthesis on eriodictyol and taxifolin production. For eriodictyol production, the related genes were overexpressed in Polf/T4S $\mathrm{S}_{\mathrm{x} 5} \mathrm{IHR}_{\mathrm{x} 2}$. For taxifolin production, the related genes were overexpressed in Po1f/T4S $\mathrm{S}_{\mathrm{x} 5} \mathrm{IHR}_{\mathrm{x} 2} \mathrm{H}$. + referred to the presence of gene overexpression. - referred to the absence of gene overexpression. 
781

782

a

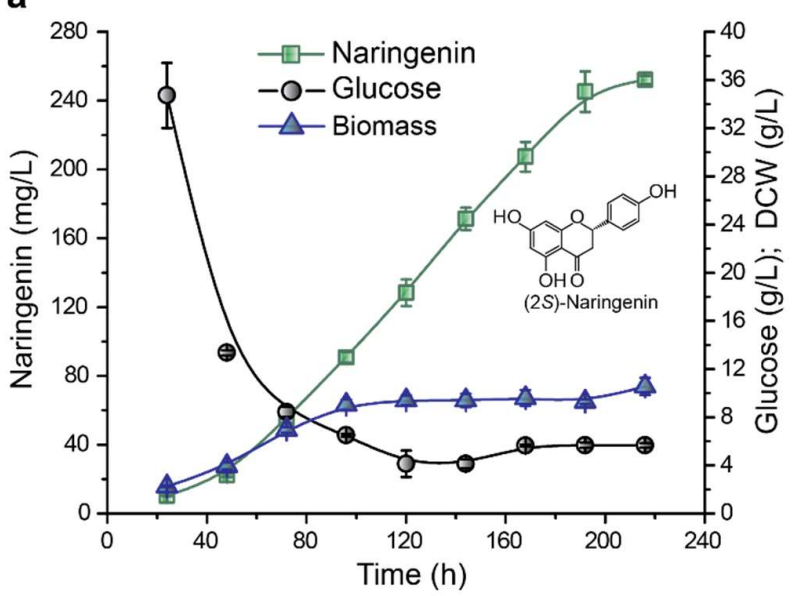

b

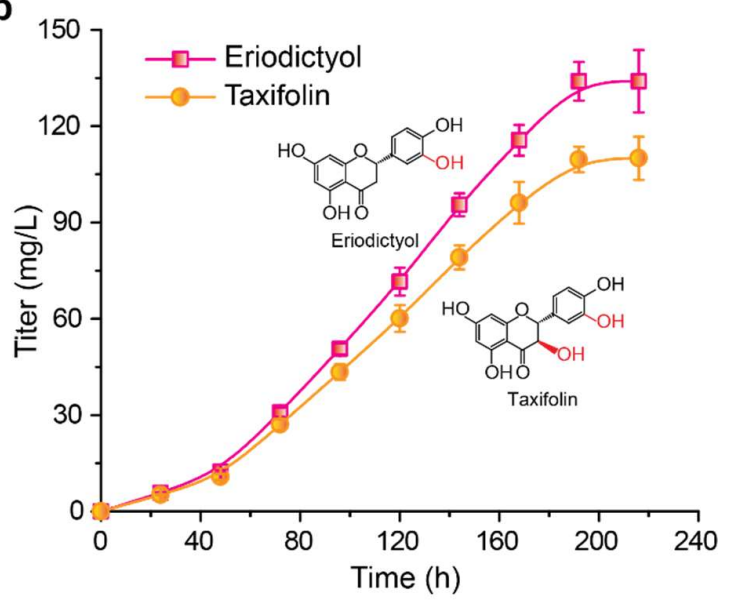

783

784 Fig. 6. Naringenin, eriodictyol and taxifolin production under the optimal conditions. (a).

785 Naringenin production; (b) Eriodictyol and taxifolin production. The engineered strains were

786 cultivated in fed-batch fermentation and buffered with $40 \mathrm{~g} / \mathrm{L} \mathrm{CaCO}_{3}$. A final concentration of 1

$787 \mathrm{mg} / \mathrm{L}$ cerulenin was supplemented at $48 \mathrm{~h}$ to inhibit fatty acid synthesis. 